\title{
Assessing physical activity in the daily life of cystic fibrosis patients
}

\author{
André Dias a,b,c,*, Lukas Gorzelniak ${ }^{\mathrm{b}, \mathrm{f}}$, Rudolf A. Jörres ${ }^{\mathrm{d}}$, Rainald Fischer ${ }^{\mathrm{e}}$, \\ Gunnar Hartvigsen ${ }^{\mathrm{a}, \mathrm{c}}$, Alexander Horsch ${ }^{\mathrm{b}, \mathrm{c}, \mathrm{g}}$ \\ ${ }^{a}$ Norwegian Center for Integrated Care and Telemedicine, University Hospital of North Norway, Tromsø, Norway \\ ${ }^{\mathrm{b}}$ Institut für Medizinische Statistik und Epidemiologie, Technische Universität München, Germany \\ ${ }^{\mathrm{c}}$ Department of Computer Science, University of Tromsø, Tromsø, Norway \\ ${ }^{\mathrm{d}}$ Institut und Poliklinik für Arbeits-, Sozial- und Umweltmedizin, LMU, Munich, Germany \\ e Medizinische Klinik Innenstadt, LMU, Munich, Germany \\ ${ }^{\mathrm{f}}$ Institute for Epidemiology, HelmholtzZentrum münchen, German Research Center for Environmental Health, Germany \\ ${ }^{g}$ Department of Clinical Medicine, University of Tromsø, Norway
}

\section{A R T I C L E I N F O}

Article history:

Available online 11 August 2012

\section{Keywords:}

Cystic fibrosis

Physical activity

Accelerometer

Exacerbation

Usability

\begin{abstract}
A B S T R A C T
Physical Activity (PA) plays an important role in the health and quality of life of cystic fibrosis (CF) patients, but little is known about their PA in daily living. With the use of accelerometers it is now possible to monitor activity profiles in detail. The goals of this study are to assess feasibility and acceptance of a longer-term use of accelerometers in daily living in CF patients, study the possibility of detecting changes in PA in relation to the patients' clinical state and compare the findings between a CF and an age-matched healthy control group. We asked $15 \mathrm{CF}$ patients to wear two accelerometers for 21 days and fill in a diary. Ten of them (age 21 to 40, mean 29.5 years) participated and delivered data that could be evaluated. We also recruited 10 age-matched control subjects. Data was processed for calculating usage time and features extracted to construct models of activity. The younger patients, particularly females, were concerned with fashion and style and considered wearing the sensors a challenge. Overall, the compliance of patients with $\mathrm{CF}$ seemed to be lower than reported for elder subjects in the literature. Time-series analysis of the data indicated characteristic patterns of PA over time, provided that data preprocessing and noise-filtering had been optimized. Further studies have to assess whether the continuous recording of PA yields additional clinical information in CF and in particular, whether it is possible to detect or even predict exacerbations in patients with CF or other diseases.
\end{abstract}

(C) 2012 Elsevier B.V. All rights reserved.

\section{Introduction}

Cystic fibrosis (CF) is a hereditary disease that affects the whole body causing progressive dysfunction of several organs that ultimately leads to premature death. Approximately 1 in 25 individuals of European descent carry the CF allele as heterozygotes. In accordance with the allele frequency, disease incidence is about 1:2500 births [1]. There are many genetic variants of the CF disorder and significant effects of the genetic environment that are associated with differences in severity and phenotype of the disease.

In CF patients, dysfunction of the CF Transmembrane Regulator (CFTR) protein creates disturbances in chloride transport through the epithelium, resulting in dehydration of the mucosal surfaces. The subsequent formation of dense mucus favors

\footnotetext{
* Correspondence to: Department of Computer Science, University of Tromsø, 9037 Tromsø, Norway. Tel.: +47 91245569.

E-mail address: andre.dias@uit.no (A. Dias).
} 
the development and persistence of bacterial infections. This in particular leads to a deterioration of pulmonary function, including breathing difficulties. Pulmonary causes are responsible for more than two thirds of all CF-related deaths [1]. CF is diagnosed in males and females with the same frequency, but for reasons that remain unclear, males tend to have a longer life expectancy [2]. According to the CF Foundation, in the United States the average life expectancy for infants born with CF in 2008 is 37.4 years [3]. One of the most important clinical events for patients with CF is an acute pulmonary exacerbation, with different causes, namely microbial infections and environmental factors. Such episodes reduce significantly the quality of life with symptoms that include severe breathing difficulty.

Physical activity (PA) is influenced by a multitude of psychological and physiological factors, including exercise capacity. Just this integrative role appears to render it suited as an overall measure of the health state. In chronically ill patients, maintaining PA as far as possible appears to be important for maintaining physical reserves but also to improve social contacts and self-esteem [4]. As, conversely, a subject's health status can be affected by the capability for PA [5], PA could even be an additional subject of intervention.

PA and associated fitness levels seem to be particularly important in CF, [6], as patients with higher aerobic capacity have higher life expectancy [7], aerobic capacity correlates with quality-of-life [8], and in adults with CF professional achievements are related to physical fitness [9]. So far, measurements of PA in CF patients have typically been limited to short recording periods of under a week; to our knowledge, there is only one study with a 2-week time frame.

Troosters et al. [10] used the Sensewear device (Body media, Pittsburgh, USA) in 20 CF patients and 20 healthy control individuals over 5-7 days, to study skeletal muscle weakness and PA. Their results indicate a contribution of physical activity to exercise tolerance, which is a crucial aspect for the definition of rehabilitation programs. Hebestreit et al. [6] monitored 7 days of PA, using the MTI/CSA 7164 accelerometer (MTI Health Services, Fort Walton Beach, USA), and correlated the results with exercise capacity. They found that both moderate and vigorous PA and average daily accelerometer count independently explained part of the variance in maximal oxygen uptake, contributing to the understanding of the importance of rehabilitation programs in CF. Fournier et al. [11] recruited 15 CF patients, in whom they measured PA for 7 days, and correlated the results with pulmonary function and exercise tolerance. To study energy expenditure in CF patients during Pseudomonas aeruginosa-related pulmonary exacerbation, Béghin et al. [12] assessed PA in 16 children, but only for $24 \mathrm{~h}$, which included a normal day at school. In this group they found no significant decrease of PA in the time between administrations of two intravenous antibiotic therapies. Wells et al. [13] evaluated the output of the Actigraph accelerometer over two weeks in 14 patients and compared the data with the habitual activity estimation scale (HAES) and a diary, concluding that a questionnaire is a reasonable instrument for estimation of PA levels in patients with CF.

Given the clinical impact of CF [1], objective measurement of PA appears to be an under explored research topic. Objective monitoring and reporting of PA can be a significant source of motivation for patients to keep a regular exercise program. It is also possible that PA could play a role in the prediction of exacerbations in CF, similar to COPD [14,15]. Exacerbations in both diseases are associated with reduced breathing capacity and consequent reduced PA level. This would require patients to wear sensors for extended periods of time or even all the time. Although the extension of monitoring is currently restricted by factors such as battery and memory capacities of the devices, this could be technically handled in the future. If we can anticipate exacerbations we can raise warnings before the event allowing the patient to prepare and avoid some of the complications. This awareness can potentially reduce the number of admissions to the hospital, as well disrupt the vicious circle where patients reduce their exercise because they are afraid of the complications during exacerbation episodes but by doing so speed up their disease progression. Overall long-term monitoring of PA can be a motivation factor to maintain or increase PA levels and thus improve disease management.

Based on these considerations, the goal of this study was three-fold: (1) to assess feasibility and acceptance of a longerterm use of accelerometers in daily living in CF patients; (2) to study the possibility of detecting changes in PA in relation to the patients' clinical state; (3) to compare the findings between a CF and an age-matched healthy control group.

\section{Methods}

This study was a pilot study aiming at feasibility and acceptability. Besides wearing two sensors for recording PA, the participants were also asked to answer a short questionnaire three times a day. The questionnaire focused on the perceived health status, traveling outside the area of residence, and the medication taken.

\subsection{Subjects}

In cooperation with the CF Unit of the Inner City Clinic of the Ludwig-Maximilians-University Munich we asked 15 $\mathrm{CF}$ out-patients to participate in the study. The interview took place during their routine consultation with their doctor. Only patients with age $>18$ years and in a stable clinical condition were included. Participation was voluntary, with no compensation. A written informed consent was obtained from each participant, and the Ethics Committee approved the study protocol. Additionally to the CF patients we recruited 10 age-matched healthy subjects without a known history of disease or condition influencing their PA as control subjects. In order to provide useful feedback to the patients and to increase their motivation to participate in the study, the acquired data were offered to inform the patients about potential findings indicating a restless leg syndrome. An overview of the participating CF patients' health status is given in Table 1. 
Table 1

Subjects' characteristics $\left(\mathrm{FEV}_{1}=\right.$ forced expiratory volume in one second, $\mathrm{PaO}_{2}=$ arterial oxygen pressure, $\mathrm{PaCO}$ arterial pressure for carbon dioxide, \%pred = values as percent of predicted (normal) values).

\begin{tabular}{llllllll}
\hline ID & Age & Height $(\mathrm{cm})$ & Weight $(\mathrm{kg})$ & $\mathrm{FEV}_{1}$ & $\mathrm{FEV}_{1} \%$ pred. & $\mathrm{PaO}_{2}$ & $\mathrm{PaCO}_{2}$ \\
\hline P1 & 40 & 175 & 66 & 1.49 & 39 & 72.2 & 38.1 \\
P2 & 25 & 179 & 67 & 3.11 & 81 & 83.0 & 33.4 \\
P3 & 26 & 166 & 50 & 1.95 & 51 & 94.0 & 41.0 \\
P4 & 21 & 160 & 61 & 4.34 & 119 & 86.4 & 37.2 \\
P5 & 26 & 184 & 74 & 1.91 & 41 & 75.0 & 40.3 \\
P6 & 31 & 180 & 55 & 3.27 & 75 & 84.3 & 40.2 \\
P7 & 30 & 157 & 49 & 2.13 & 75 & 77.0 & 33.3 \\
P8 & 35 & 190 & 85 & 4.14 & 89 & 76.8 & 37.6 \\
P9 & 36 & 169 & 62 & 1.69 & 46 & 50.2 & 40.3 \\
P10 & 25 & 160 & 57 & 1.42 & 39 & 68.0 & 42.1 \\
\hline Mean & 29.5 & 172 & 62.6 & 2.55 & 65.5 & 76.7 & 38.4 \\
\hline
\end{tabular}

\subsection{Devices}

To obtain information on different aspects of movement, we used the uniaxial accelerometer Actigraph GT1M (Actigraph LLC, Pensacola, Florida, USA), placed at the ankle, and the tri-axial accelerometer RT3 (Stayhealthy Inc. Elkader, Iowa, USA) placed at the hip. Both sensors have been technically validated in other studies [16,17]. The sensors were configured to record activity once a minute. The GT1M provides information on the acceleration in the vertical axis, by sampling movements at $30 \mathrm{~Hz}$ and integrating over $1 \mathrm{~min}$. The RT3 provides a Vectorial Measurement Unit (VMU), which is the vectorial sum of the data collected for the 3 axes.

\subsection{Acquisition and handling of sensor data}

Given the frequency of exacerbations in CF patients, a measurement period of 21 days was chosen. In 10-15 patients this implied 210-315 study days and thus a great likelihood to observe at least 1 or 2 deteriorations of the disease. In order to raise the likelihood of exacerbations in the patient group, we performed the study during early spring time.

The subjects were given a brief demonstration of the placement and use of the two sensors. They were asked to wear the hip sensor during their daily living activities, from getting up in the morning until going to sleep at night, always on the dominant side of the body, in the clip provided by the manufacturer to avoid slippage. For the ankle sensor they were asked to wear it $24 \mathrm{~h}$ a day, as long as it was comfortable. To avoid slippage of the ankle sensor we used elastic belts with clippers. By the end of the measurement period, questionnaires and sensors were returned by post in a postage-paid and addressed envelope.

According to the specification of the GT1M sensor, the battery lasts for 21 days. Despite this, to avoid the sensor stopping recording we provided a USB charger and cable to the participants and reminded them by phone after 10-12 days to recharge the sensor. Upon arrival at the laboratory, sensors and questionnaires were checked, and data were collected and exported to a spreadsheet using the software provided by the manufacturers.

\subsection{Data analysis}

To assess compliance we calculated the percentage of time over which each patient had used the sensor as defined by the algorithm proposed by Hecht et al. [18] and described in Fig. 1. For the RT3 sensor we used a threshold of 5 VMU [18], given the fact that this sensor produces noise under stationary conditions. For the GT1M sensor we defined a threshold of 0 activity counts, given its insensitivity to noise under stationary conditions. These data were used to define, whether the sensor was worn at a given time or not (Fig. 1). We did not control for readings due to external movement such as riding a car or bike.

To model PA profiles data was excluded from $10 \mathrm{pm}$ to $6 \mathrm{am}$, as we had a low volume of usable data for this period. From this data we extracted 8 features from a scatter plot of the filtered values measured by each sensor. In the scatter plot used for this, consecutive values were plotted against each other. For each two consecutive activity measurements $X_{i}$ and $X_{i+1}$, at minute $i$ and $i+1, X_{i}$ is the coordinate on the $x$-axis and $X_{i+1}$ is the coordinate on the $y$-axis. The features evaluated comprised: coordinates of the centroid of all points (median coordinate along $X$ and $Y$ axis), proportion of points in $5 \%$ of the area around the centroid (percent relative to maximum area defined by the signal amplitude), density of points in each of the four quadrants (also defined by the maximum area of the signal amplitude) and average distance of points to the centroid. We choose these features after comparison of all the scatter plots and identifying visual distinctions between patients and controls. These features were used as input for a feed-forward neuronal network (NN) with 20 hidden neurons. The NN was trained to discriminate between patients and control subjects, using the leave-one-out method, which is a standard cross-validation scheme for small data sets. 


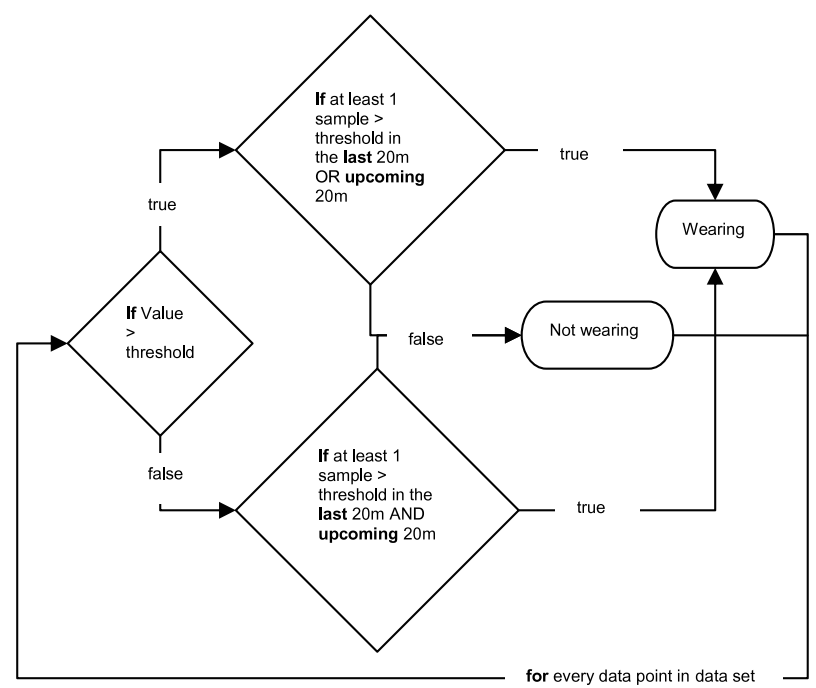

Fig. 1. Algorithm for determining if a sensor was worn at a given minute.

Table 2

Feedback from the patients about participation in the study.

\begin{tabular}{lll}
\hline Gender & Start & Recruitment remarks \\
\hline M & 24.03 & None \\
M & 24.03 & "I do not know what my girlfriend is going to think about me wearing the sensors" \\
F & Refused & "The sensors are too clumsy too wear at work" \\
F & Refused & "Cannot use red one beneath the tight trousers or the high shoes. The grey one is too visible to wear" \\
M & 24.03 & None \\
M & 25.03 & "3 weeks is too long. I will accept 1 week. Are the alarms in the shops going to start because of the sensor?" \\
F & Refused & "3 weeks is too long" \\
M & Refused & "Going for a golf tournament for 10 days. Cannot use them because of balance" \\
F & Refused & "Having other complications from asthma. Physical activity very low" \\
F & 25.03 & None \\
M & 31.03 & "I have bad sleep, so the restless leg syndrome info is very important" \\
M & 01.04 & \\
M & 02.04 & "21 days is very long" Extensive explanation of the questionnaire was needed \\
M & 07.04 & None \\
F & 07.04 & None
\end{tabular}

\section{Results}

\subsection{Acceptance and usability}

We asked 15 young patients ( 6 women) to participate in the study (Table 2 ). Of these, 10 subjects (age 21-40, mean age 29.5 years) agreed to participate (Table 1). Only two women were among them, however, when they returned the sensors we observed that one of them had not worn the devices.

The most common reason for not participating was related to fashion. Typically, women commented: "I don't want to be seen with a sensor nor shall my friends/colleagues see me wearing this" or "During winter I wear tight boots, so an anklemounted device would not fit. During summer, it would be too visible with smaller shoes or with skirts". Men had more practical reasons to reject the sensors: "I usually participate in studies. However, I have an upcoming golf tournament where these devices might disturb my balance". Among women the RT3 sensor was mentioned several times as being "ugly", "too visible" or "too big". The GT1M sensor was referred to as "too visible" due to its color, while its size was deemed acceptable.

Men seemed to be more likely to accept the sensors due to their technical aptitude and interest. Some ascribed a certain status to the hip-worn device and did not seem to be concerned about the visibility. "I used to be a fire fighter, so I am familiar with devices". "This device makes me look more important”. Moreover, male subjects raised technical questions regarding the measurement and the feedback. Independent of gender, the main reasons for refusing were due to the burden of wearing sensors or the long period of measurement. "I would do it for a few days, but 3 weeks is way too long".

Some patients complained about the usability of the straps provided for the GT1M sensor. Especially the hard plastic parts caused discomfort after a few hours of continuous wearing. We later replaced them with Velcro straps, in line with the manufacturer, who started to sell equivalent straps during the study period. 

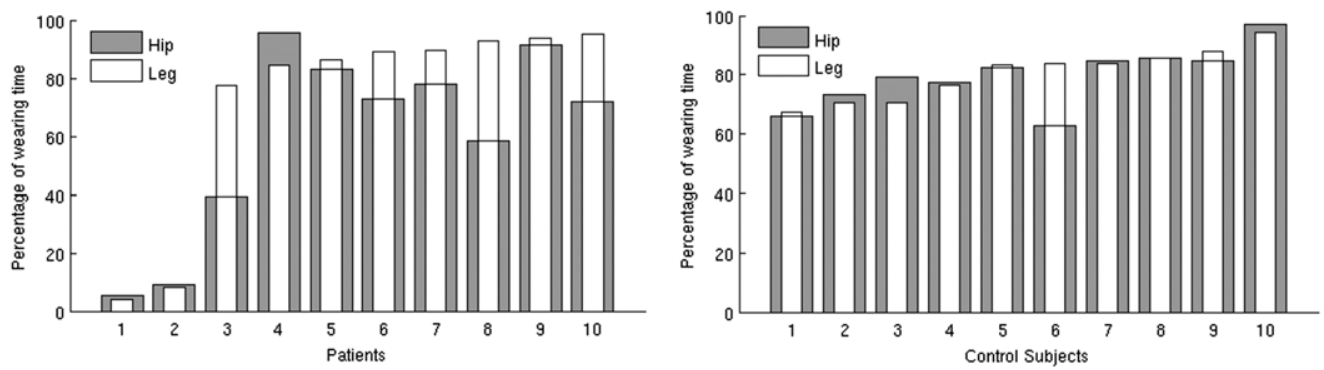

Fig. 2. Percentage of time, from 6 am to $10 \mathrm{pm}$, which patients and controls used each sensors.

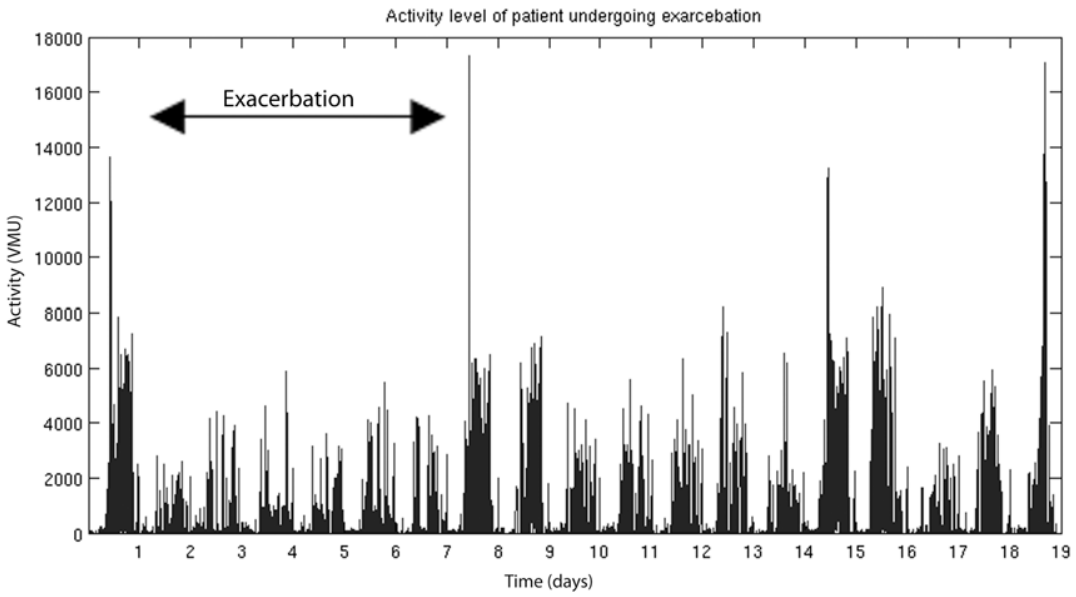

Fig. 3. Physical activity at leg, 1 min sampling, (GT1M) for a patient undergoing an exacerbation for 6 days (arrow area).

Table 3

Average sensor usage time.

\begin{tabular}{llll}
\hline & & Patients (\%) & Controls (\%) \\
\hline \multirow{2}{*}{6 am-10 pm } & Hip & 60.4 & 79.3 \\
& Leg & 72.1 & 80.3 \\
\hline \multirow{2}{*}{ All day } & Hip & 43.8 & 55.4 \\
& Leg & 58.5 & 58.3 \\
\hline
\end{tabular}

\subsection{Compliance}

Fig. 2 shows the percentage of time each patient and control subject used both sensors, during the period of 6 am-10 pm. Except for two patients who used the sensors less than 20\% of time, subjects showed a relatively high percentage of compliance. Most patients used the leg sensor more than the hip sensor. This indicates that they were interested in the restless leg syndrome diagnostics. Control subjects seemed to show no interest in this diagnostics, having similar usage percentages for both sensors.

Table 3 shows the average time of usage for all patients and control subjects, considering the whole filtered data set to include only the period $6 \mathrm{am}-10 \mathrm{pm}$. Control subjects showed better compliance in using the sensors, but the results for the patient group were skewed due to the two patients with a very low percentage of usage. The compliance for the hip sensor was poor, even if the night periods were excluded. This seems to indicate the initial skepticism of patients as regards the size and visibility of the sensor. The percentages were significantly lower than those reported by Hecht et al. [18] for COPD.

\subsection{Physical activity in relation to exacerbations}

We decided to take into consideration the prevalence of exacerbations in $\mathrm{CF}$ when designing the study protocol. In fact one exacerbation episode was detected in one of the (clinically relatively well-controlled and stable) CF patients. The exacerbation was reported by the participant in the questionnaire but did not lead to hospital admission.

Fig. 3 shows the PA time series for this patient, where the exacerbation period is marked. Unfortunately, PA had not been measured for a long time in the days preceding the exacerbation. Nevertheless there appeared to be a prolonged drop in the 

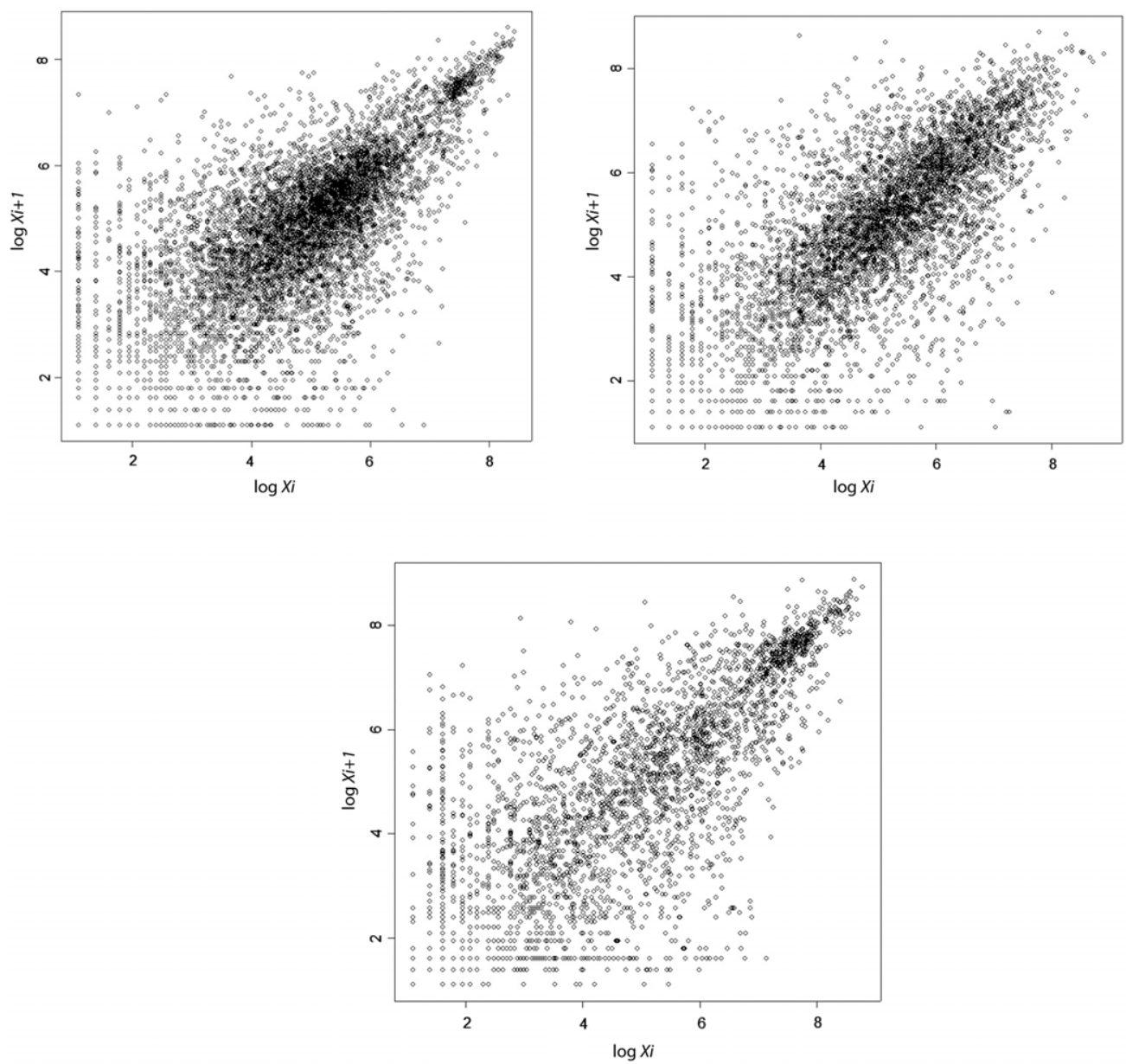

Fig. 4. Scatter plots of leg sensor (GT1M); each point represents two consecutive measures; data for two CF patients (top row) and a control subject (bottom row) is shown.

activity level during the exacerbation episode irrespective of the shorter drops seen afterwards. This renders it a sensible aim to further explore the potential of using PA as an early predictor of exacerbations.

\subsection{PA profile modeling}

Several techniques to model the individual patients' PA profiles were explored. As an example, Fig. 4 shows an exploratory scatter plot of the distance between two consecutive measurements, from two CF patients and one control subject. These plots indicate distinct movement profiles between subjects and even hint at a distinction between CF patients and controls. They show the patterns of physical activity change at $1 \mathrm{~min}$ intervals. Higher density of points around a central point indicates a smooth pattern of movement with low amplitude changes along time. On the other hand, sparse plots indicate highly dynamic changes of activity. The location of the highest density cluster along the diagonal indicates the overall intensity of PA.

The NN aiming to discriminate between CF patients and control subjects could be successfully trained by the filtered leg sensor data, achieving an accuracy of $85 \%$, with sensitivity of $80 \%$ and specificity of $90 \%$. The $F$-score for this setup was $84 \%$ and the Area Under the Curve (AUC) of $81 \%$. By contrast the NN trained with data from the hip sensor achieved an accuracy of $63 \%$, with sensitivity of $65 \%$ and specificity of $70 \%$. The confusion matrix for the NN trained with leg data is shown in Table 4.

\section{Discussion}

The long-term use of accelerometers in young, chronically ill patients poses a number of challenges. Apparently, young subjects are more concerned about fashion and personal appearance. Therefore, strange-looking devices are not welcome in their daily life. We identified a gender dependence of the acceptability of such devices that has not been widely reported, but which seems to be evident in several studies. The color and positioning of the devices were clearly a major drawback. 
Table 4

Confusion matrix for PA modeling using a neuronal network after training based on the 8 features of the leg sensor data to discriminate between control subjects and patients.

\begin{tabular}{lccl}
\hline & Patients & Controls & Sum \\
\hline NN predicted patient & 8 & 1 & 10 \\
NN predicted control & 2 & 9 & 10 \\
Sum & 10 & 10 & 20 \\
& & Accuracy & $85 \%$ \\
& & Sensitivity & $80 \%$ \\
& & Specificity & $90 \%$ \\
\hline
\end{tabular}

Women showed less willingness to wear devices, citing reasons of fashion or comfort. Men, on the other hand, used technical proficiency and social status arguments as reasons to accept the disturbance of wearing these fairly uncomfortable devices. These usability issues seem to be confirmed by the lower compliance rate for this group as compared to elder patients. The RT3 sensor, possibly because of the old-fashioned design, had slightly shorter wearing time than the GT1M. Our observations point towards the need for inconspicuous devices if these should be implemented in the long-term monitoring of patients in future. They also suggest that devices placed at the ankle might face acceptance problems.

Reasonable sensitivity and specificity in distinguishing patients from control subjects was achieved, when conducting simple PA modeling with subsequent use of a neuronal network. Our results indicate that the data collected by the leg sensor is more robust for this task. We used a small set of simple features as input for the NN, inspired by the scatter plot of the data. Many other types of features have been used and proposed for the analysis of accelerometer data. Large sets of heterogeneous features [19] and other instances inspired on frequency domain [20] or time-scale analysis [21]. Such applications focus on short-term monitoring. To our knowledge this is the first use of a classification system for data from long-term monitoring. Our results with this small set of simple features lead us to believe that further work on more robust features may increase the capacity of classification.

However, the small sample size of this pilot study did not allow us to draw further conclusions. Naturally, the clinically interesting application is not the distinction from healthy subjects, but the individual characterization of patients as well as the possibility to detect changes over time, possibly supported by an individually trained neuronal network implemented into the activity monitor.

Correspondingly, the detection or prediction of exacerbations in CF patients remains an issue for further studies. However, the data in one of the patients seemed promising, although we had missed the period prior to the exacerbation from recording. Future studies must consider longer periods of recording and a larger study group to be able to cover a significant number of exacerbations.

We would like to emphasize that the use of PA recording for the purpose of exacerbation prediction in patients with CF or COPD seems a very reasonable approach for future research, given the unreliability of lung function recording at home and the non-objectivity of symptoms. From clinical studies and common experience it is well known that physical capacity and activity are associated with the clinical state, in particular exacerbations [15]. Data from accelerometers should be explored for not only linear changes of physical activity before and during exacerbation, but also exploring other dimensions of the time series, such as frequency or scale. The easy of use and large datasets generated make them good tools for that.

According to our results, manufacturers of accelerometer devices for medical applications should have to pay more attention to fashion-related issues and attempt to create devices which appeal to a young population, by choosing neutral or skin-like colors, trendy form factors or perhaps by integrating these sensors into commonly used devices such as mobile phones. The success of Nike + ipod (Nike corp. Beaverton, Oregon) and Fitbit (Fitbit, San Francisco, California) products, focused on the sports market, should be taken into consideration when designing sensors for medical applications. We suggest that this can raise the acceptance of usage in such groups to a reasonable percentage, with the consequence that long-term, quasi-continuous recording becomes feasible.

\section{Acknowledgments}

This research was funded/supported by the Graduate School of Information Science in Health (GSISH) and the TUM Graduate School. André Dias is also supported by the Portuguese Foundation for Science and Technology (FCT), by scholarship SFRH/BD/39867/2007 and Research Council of Norway Grant No. 174934.

\section{References}

[1] J. Blic, M. Le Bourgeois, D. Hubert, Mucoviscidose, in: Editions Scientifiques et Medicales, Vol. 5, Elsevier, 2001, pp. 1-14.

[2] M. Rosenfeld, R. Davis, S. FitzSimmons, M. Pepe, B. Ramsey, Gender gap in cystic fibrosis mortality, Am. J. Epidemiol. 145 (9) (1997) 794-803.

[3] Cystic Fibrosis Foundation. Cystic fibrosis foundation patient registry 2008 annual data report. Bethesda, Maryland: Cystic Fibrosis Foundation, 2008.

[4] H. Kolberg, Cystic fibrosis and physical activity: an introduction, Int. J. Sports Med. 9 (Suppl. 1) (1988) 2-5.

[5] S. Spencer, P.M. Calverley, P. Sherwood Burge, P.W. Jones, ISOLDE study group inhaled steroids in obstructive lung disease. Health status deterioration in patients with chronic obstructive pulmonary disease, Am. J. Respir. Crit. Care. Med. 163 (1) (2001) 122-128. 
[6] H. Hebestreit, S. Kieser, S. Rudiger, T. Schenk, S. Junge, A. Hebestreit, et al., Physical activity is independently related to aerobic capacity in cystic fibrosis, Eur. Respir. J. 28 (4) (2006) 734-739.

[7] P.A. Nixon, D.M. Orenstein, S.F. Kelsey, C.F. Doershuk, The prognostic value of exercise testing in patients with cystic fibrosis, N. Engl. J. Med. 327 (25) (1992) 1785-1788.

[8] D.M. Orenstein, P.A. Nixon, E.A. Ross, R.M. Kaplan, The quality of well-being in cystic fibrosis, Chest 95 (2) (1989) 344-347.

[9] D.D. Frangolias, C.L. Holloway, S. Vedal, P.G. Wilcox, Role of exercise and lung function in predicting work status in cystic fibrosis, Am. J. Respir. Crit Care. Med. 167 (2) (2003) 150-157.

[10] T. Troosters, D. Langer, B. Vrijsen, J. Segers, K. Wouters, W. Janssens, et al., Skeletal muscle weakness, exercise tolerance and physical activity in adults with cystic fibrosis, Eur. Respir. J. 33 (1) (2009) 99-106.

[11] C. Fournier, L. Bosquet, S. Leroy, T. Perez, R. Neviere, B. Wallaert, Measurement of daily physical activity in patients with cystic fibrosis, Rev. Mal. Respir. 22 (1 Pt 1) (2005) 63-69.

[12] L. Béghin, F. Gottrand, L. Michaud, H. Vodougnon, N. Wizla-Derambure, R. Hankard, et al., Energetic cost of physical activity in cystic fibrosis children during Pseudomonas aeruginosa pulmonary exacerbation, Clin. Nutr. 24 (1) (2005) 88-96. (Edinburgh, Scotland).

[13] G.D. Wells, D.L. Wilkes, J. Schneiderman-Walker, M. Elmi, E. Tullis, L.C. Lands, et al., Reliability and validity of the habitual activity estimation scale (HAES) in patients with cystic fibrosis, Pediatr. Pulmonol. 43 (4) (2008) 345-353.

[14] T. Troosters, R. Casaburi, R. Gosselink, M. Decramer, Pulmonary rehabilitation in chronic obstructive pulmonary disease, Am. J. Respir. Crit. Care. Med. $172(1)(2005) 19-38$.

[15] H.Q. Nguyen, B. Steele, J.O. Benditt, Use of accelerometers to characterize physical activity patterns with COPD exacerbations, Int. J. Chron. Obstruct. Pulmon. Dis. 1 (4) (2006) 455-460.

[16] R.M. Boon, M.J. Hamlin, G.D. Steel, J.J. Ross, Validation of the New Zealand physical activity questionnaire (NZPAQ-LF) and the international physical activity questionnaire (IPAQ-LF) with accelerometry, Br. J. Sports Med. (2008).

[17] M.P. Rothney, E.V. Schaefer, M.M. Neumann, L. Choi, K.Y. Chen, Validity of physical activity intensity predictions by actigraph, actical, and RT3 accelerometers, Obesity 16 (8) (2008) 1946-1952.

[18] A. Hecht, S. Ma, J. Porszasz, R. Casaburi, COPD clinical research network. methodology for using long-term accelerometry monitoring to describe daily activity patterns in COPD, COPD 6 (2) (2009) 121-129.

[19] J. Lester, T. Choudhury, N. Kern, G. Borriello, B. Hannaford, A hybrid discriminative/generative approach for modeling human activities, in: Proceedings of the 19th International Joint Conference on Artificial Intelligence, IJCAI'05, Morgan Kaufmann Publishers Inc., San Francisco, CA, USA, 2005, pp. 766-772.

[20] T.M.E. Nijsen, R.M. Aarts, P.J.M. Cluitmans, P.A.M. Griep, Time-frequency analysis of accelerometry data for detection of myoclonic seizures, IEEE Trans. Inf. Technol. Biomed. 14 (5) (2010) 1197-1203.

[21] M. Sekine, M. Akay, T. Tamura, Y. Higashi, T. Fujimoto, Fractal dynamics of body motion in patients with Parkinson's disease, J. Neural Eng. 1 (1) (2004) 8-15. 